\title{
IRS-Assisted Communications Under Practical Channel Estimation and Hardware Model
}

\author{
Lucas C. de P. Pessoa, Gilderlan T. de Araújo, Paulo R. B. Gomes and André L. F. de Almeida
}

\begin{abstract}
In wireless communications, the propagation environment is not always favorable and may have several adverse conditions for data transmission and reception. The improper propagation conditions can be mitigated by the operator or by the current signal processing techniques. This classical paradigm can be rethought with the emerging concept of intelligent reflecting surface (IRS). IRS is a cost-effective, power-efficient and low hardware complexity solution capable of make wireless propagation environments more favorable by controlling in a software-defined way the amplitude and phase of the incident signal in order to improve the signal-to-noise ratio or mitigate interference at the intended receiver. However, in practice, the IRS operation are subject to inevitable impairments and practical issues, such as hardware limitations, blockages, and channel estimation errors that directly affects the system performance. In this paper, we study an IRS-assisted wireless communication system operating under one practical hardware model, which blockages at the IRS reflecting units are taken into account. Then, we investigate the impact of these real-world impairments on the system performance under practical channel estimation. The performance of an IRS-assisted communication under such practical constraints are evaluated in terms of the spectral efficiency (SE), normalized mean square error (NMSE) and symbol error rate (SER).
\end{abstract}

Keywords-Intelligent reflecting surface, varactor model, blockages, channel estimation, spectral efficiency.

\section{INTRODUCTION}

Intelligent Reflecting Surface (IRS) has attracted recent attention of researchers and industry. With this new concept, many efforts have been made to investigate the real benefits and limitations of this promising technology in the context of wireless communications [1]. This is motivated in part by the nature of the IRS, which is composed of low-cost semipassive reflecting units capable of "reprogramming" the channel by self-adjusting the amplitude and phase of the incident signal, enabling the concept of smart wireless environment. In addition, IRS is capable of boosting the current wireless network performance by providing greater spectral and energy efficiency gains.

Several works have studied the performance of IRS-assisted wireless communication systems considering different sce-

Lucas C. de P. Pessoa, Gilderlan T. de Araújo, Paulo R. B. Gomes and André L. F. de Almeida are with the Wireless Telecommunications Research Group (GTEL), Department of Teleinformatics Engineering (DETI), Federal University of Ceará (UFC), Fortaleza, Brazil. E-mails: \{lucascampos, gilderlan, paulo, andre\}@gtel.ufc.br. This work was supported by the Ericsson Research, Sweden, and Ericsson Innovation Center, Brazil, under UFC. 48 Technical Cooperation Contract Ericsson/UFC. This study was financed in part by the Coordenação de Aperfeiçoamento de Pessoal de Nível Superior - Brasil (CAPES)-Finance Code 001, and CAPES/PRINT Proc. 88887.311965/2018 00. André L. F. de Almeida like to acknowledge CNPq for its financial support under the grant 306616/2016-5. narios and useful cases. The authors of [2] have analyzed the application of IRS in unmanned aerial vehicle (UAV) communication. Based on game theory, a dynamic network service selection was proposed in [3]. Furthermore, in [4], the IRS has been integrated into a satellite-terrestrial network to enable a secure cooperative transmission. The authors of [5] present the potentialities of an IRS-assisted smart radio, envisioning $6 \mathrm{G}$ networks and beyond. However, the performance gains achieved in IRS-assisted systems strongly depends of the channel estimation accuracy, as well as the hardware technology considered to implement the reflective units. First, several works have focused on the channel estimation problem, and different solutions have been proposed in the literature. The first work that tackles this problem assumed an idealized IRS model, where the reflecting units have an infinite phase shift resolution and an ideal (unitary) amplitude response [6]. Some works have adopted a more practical view on the IRS model by considering hardware limitations. In [7], the authors consider an IRS operating under finite resolution of phase shifts, where the reflecting units can be tuned from a finite number of phases. In addition, the paper [8] have studied constraints on the amplitude response of the reflecting units. The authors in [9] have considered an IRS model under blockages. In this case, the IRS operates under failures of some reflecting units, for instance, due to uncontrollable environmental circumstances or atmospheric effects.

Although the works mentioned have studied some practical aspects related to the IRS model, the performance impact of physical (circuit-based) hardware models combined with actual channel estimation deserves attention and can be better understood. In particular, the response of the IRS is limited by the hardware technology and, more specifically, by the parameters of the electric circuits that make up the reflecting units. Currently, the most popular hardware model for IRS is based on varactors [10] and PIN diodes [11], although liquid crystals have also been considered [12]. Another recently studied model is based on graphene technology, which can have a great appeal in the terahertz band [13]. Hence, the IRS response depends directly on its physical design, since the different construction mechanisms imply different restrictions that affect the overall communication performance. In this paper, we combine several realistic aspects such as channel estimation errors, practical hardware model and blockages in the IRS reflecting units and study their effects on the system performance. For simplicity, we consider a single-input singleoutput (SISO) IRS-assisted wireless communication system and we use the most popular approach for channel estimation, known as the ON/OFF method [14]. 


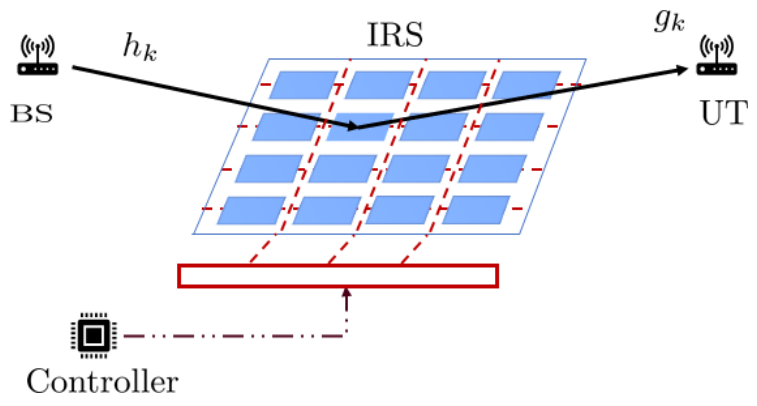

Fig. 1. IRS-assisted communication system.

Notation: Scalars and column vectors are represented by lowercase letters $(a, b, \ldots)$ and boldface lowercase letters $(\mathbf{a}, \mathbf{b}, \ldots)$, respectively. The transposition of a given vector $\mathbf{a}$ is denoted by $\mathbf{a}^{\mathrm{T}}$. The operator $\odot$ denotes the Hadamard product. $|x|$ and $\not x$ denote the absolute value and the phase of the complex-valued scalar $x$, respectively. The Frobenius norm of $\mathbf{a}$ is represented by $\|\mathbf{a}\|_{\mathrm{F}}$.

\section{SySTEM MODEL}

We consider a single-antenna transmitter and receiver communicating system with an assistance of an IRS composed of $K$ reflecting units along with a smart controller, as illustrated in Figure 1. We assume that some IRS units can be fully blocked, i.e., $K_{B}=K \cdot P_{B}$ random units at the IRS are subject to blockages, where $P_{B}$ denotes the blockage occurrence probability. We assume a downlink communication, where the transmitter is the base station (BS) and the receiver is the user terminal (UT). However, our signal model also applies to the uplink case by just inverting the roles of the transmitter and the receiver. The direct channel between the BS and the UT is assumed to be unavailable. Assuming a flat fading channel, the signal received at the UT can be expressed as follows

$$
y=\left[\sum_{k=1}^{K} h_{k} e_{k} a_{k} s_{k} g_{k}\right] x+n,
$$

where $h_{k}$ and $g_{k}$ are the Rayleigh fading channels between the BS and the IRS, and between the IRS and the UT via the $k$-th IRS unit $(k=1, \ldots, K), x$ denotes the pilot signal, and $n$ is the additive white Gaussian noise (AWGN) term with variance $\sigma^{2}$. The received signal in (1) can be rewritten in vector form as follows

$$
y=\mathbf{g}^{\mathrm{T}} \operatorname{diag}(\mathbf{e} \odot \mathbf{a} \odot \mathbf{s}) \mathbf{h} x+n,
$$

where $\mathbf{h}=\left[h_{1}, \ldots, h_{K}\right]^{\mathrm{T}} \in \mathbb{C}^{K \times 1}$ and $\mathbf{g}=\left[g_{1}, \ldots, g_{K}\right]^{\mathrm{T}} \in$ $\mathbb{C}^{K \times 1}$ are the vectors of channel coefficients between the BS-IRS and IRS-UT, respectively. The $k$-th entry of $\mathbf{e}=$ $\left[e_{1}, \ldots, e_{K}\right] \in \mathbb{R}^{K \times 1}$ models the blockage of the corresponding IRS unit. Throughout this paper, we assume that the entries of e follows a Bernoulli distribution, i.e., $e_{k}=0$ if the $k$ th IRS unit is blocked and $e_{k}=1$ otherwise $^{1}$. The vectors $\mathbf{a}=\left[a_{1}, \ldots, a_{K}\right] \in \mathbb{R}^{K \times 1}$ and $\mathbf{s}=\left[e^{j \phi_{1}}, \ldots, e^{j \phi_{K}}\right] \in \mathbb{C}^{K \times 1}$ contain the IRS amplitude responses and the phase shifts.

\footnotetext{
${ }^{1}$ We can also extend this analysis for different levels of blockages. For instance, we can assume that the IRS units are partially blocked. In this case, the entries of $\mathbf{e}$ are uniformly distributed between 0 and 1 .
}

Note that the received signal in (2) unifies in its structure different kinds of practical impairments on the IRS model. In other words, the vector e models the presence of possible blockages, while a and $\mathbf{s}$ model the amplitude and phase shift constraints of the considered hardware design. In the following, we discuss the most common signal models in the literature as particular cases of Equation (2).

\section{A. Idealized IRS Model}

In the ideal case, the IRS reflecting units have a perfecting functioning, since practical amplitude and phase impairments are neglected [8]. The phase shifts have infinity resolution, which means that $\phi_{k}$ can assume any value within the range $(0,2 \pi) \forall k$. The amplitude response is always unitary, i.e., $a_{k}=1 \forall k$, and the IRS units are not blocked, i.e., $e_{k}=1$ $\forall k$. Based on these assumptions, the received signal in (2) simplifies to

$$
y=\mathbf{g}^{\mathrm{T}} \operatorname{diag}(\mathbf{s}) \mathbf{h} x+n .
$$

This signal model corresponds to the best unfeasible scenario in terms of system operation, since the IRS is not affected by any practical impairment. Therefore, it is considered as a baseline system model for performance benchmark.

\section{B. IRS Model Under Discrete Phase Shift Resolution and Amplitude Fluctuations}

In practice, the IRS controller, e.g., an Field Programmable Gate Array (FPGA) is capable of tuning a finite number of discrete phases $\bar{\phi}_{k} \forall k$. In this case, all possible phase shifts can be uniformly distributed in the $\mathbb{F}$ set as follows [7]

$$
\mathbb{F}=\{0, \Delta \phi, \ldots,(F-1) \Delta \phi\} \quad \text { and } \quad \Delta \phi=\frac{2 \pi}{F},
$$

where $F=2^{b}$ denotes the number of possible discrete phase levels and $b$ is the number of bits necessary to represent each discrete phase shift, i.e., $\bar{\phi}_{k} \in \mathbb{F} \forall k$. Assuming that the amplitude response of the reflecting units fluctuates due to power dissipation in a practical hardware, we have $0<a_{k}<$ 1 , yielding the following signal model

$$
y=\mathbf{g}^{\mathrm{T}} \operatorname{diag}(\mathbf{a} \odot \mathbf{s}) \mathbf{h} x+n,
$$

where $\mathbf{s}=\left[e^{j \bar{\phi}_{1}}, \ldots, e^{j \bar{\phi}_{K}}\right] \in \mathbb{C}^{K \times 1}$. When blockages on the IRS reflecting units are also considered, the more general received signal in (2) holds.

\section{Varactor-Based IRS Model}

The signal model discussed in the previous section considered that the amplitude responses of the IRS have a random nature and can be modeled independently from the phase shifts. Although these model provide a simpler approach to study the performance of IRS-assisted communications, they do not consider constraints imposed by a physical hardware model. There is, however, a fundamental coupling between the amplitudes and the phase shifts of the reflecting units that depend on the electric parameters of the considered hardware model. Its impact should also be taken into account when realistic impairments are studied. 


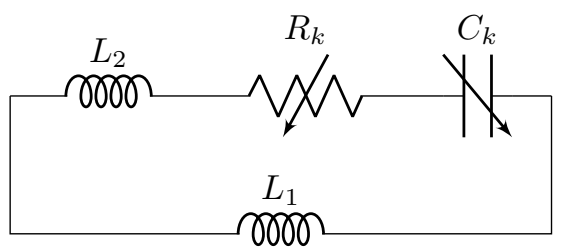

Fig. 2. Illustration of the equivalent varactor circuit model.

TABLE I

PARAMETERS USED IN THE VARACTOR MODEL

\begin{tabular}{|c|c|}
\hline Element & Value \\
\hline Impedance in free space $\left(Z_{0}\right)$ & 377 \\
\hline Indutance 1 $\left(L_{1}\right)$ & $2.5 \mathrm{nH}$ \\
\hline Indutance 2 $\left(L_{2}\right)$ & $0.7 \mathrm{nH}$ \\
\hline Effective capacitance $\left(C_{k}\right)$ & {$[0.47-2.35] p \mathrm{~F}$} \\
\hline Angular frequency $(\omega)$ & $4.8 \pi \times 10^{9}$ \\
\hline
\end{tabular}

To this end, we consider a hardware model based on varactor diodes [10], [15]. This model consists of an equivalent circuit composed of resistors, inductors and capacitors that form the parallel resonant circuit illustrated in Figure 2, whose impedance is given by

$$
Z_{k}\left(C_{k}, R_{k}\right)=\frac{j \omega L_{1}\left(j \omega L_{2}+\frac{1}{j \omega C_{k}}+R_{k}\right)}{j \omega L_{1}+\left(j \omega L_{2}+\frac{1}{j \omega C_{k}}+R_{k}\right)},
$$

where $L_{1}, L_{2}, C_{k}, R_{k}$ and $\omega$ denote the inductance, effective capacitance, effective resistance, and angular frequency of the incident signal, respectively. From (6), the reflection coefficient of the $k$-th IRS unit is modeled as

$$
v_{k}=\frac{Z_{k}\left(C_{k}, R_{k}\right)-Z_{0}}{Z_{k}\left(C_{k}, R_{k}\right)+Z_{0}},
$$

where $Z_{0}$ represents the free-space impedance. Therefore, the corresponding amplitude and phase shift response of the $k$-th IRS unit are respectively obtained from (7) as follows

$$
a_{k}=\left|v_{k}\right| \quad \text { and } \quad \phi_{k}=\not v_{k} .
$$

In practice, each IRS reflecting unit can only take a finite number of discrete phase shifts values, as mentioned in Section II-B. Therefore, the continuous phase shift $\phi_{k}$ in (8) should be rounded to its best discrete level $\bar{\phi}_{k}$ into the set $\mathbb{F}$ in (4). Based on this minimum distance criterion, $\bar{\phi}_{k}$ is chosen when

$$
\bar{\phi}_{k}=\min \left|\phi_{k}-f \cdot \Delta \phi\right|, f=1, \ldots, F-1 .
$$

To illustrate the relationship between the amplitude and phase shift at each IRS unit for different resistance values, Figure 3 depicts the amplitude vs. phase response curves for the circuit parameters shown in Table I. We can observe that the amplitude response presents different ranges of variation as a function of resistance. More specifically, by increasing the resistance, the amplitude values decrease, indicting unavoidable absorption or energy dissipation. Moreover, we can also observe that the practical varactor model in fact captures the typical behaviour of the phase-dependent amplitude variation in the IRS units. This property was neglected in the signal models of Section II, which do not consider the physical coupling between $a_{k}$ and $\phi_{k}$.

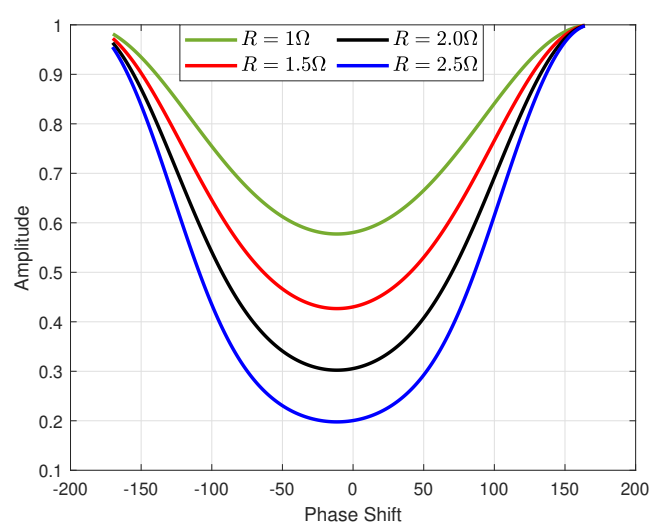

Fig. 3. Amplitude vs. phase shift coupling for a varactor-based IRS model.

\section{Channel Estimation AND IRS OptimizATION}

\section{A. Channel Estimation}

The optimization of the IRS phase shifts require the knowledge of the channel state information, which can be estimated at the receiver from pilots sent by the transmitter and reflected by the IRS. Note that a passive IRS model is assumed here, which means that the IRS does not have any digital signal processing capability. Therefore, the optimized phase shifts should be computed at the receiver from the estimated channel, and conveyed to the IRS smart controller using control signaling. Among the different channel estimation approaches in the literature, we consider the state-of-the-art ON/OFF method [14] for simplicity. It consists of sequentially estimating the channel coefficient associated with each reflecting unit by switching each individual IRS element ON and OFF. More specifically, each element of the cascaded channel is estimated at each time slot $k=1, \ldots, K$ from a pilot sent by the transmitter and reflected by the corresponding IRS unit. Since the pilot $x$ is transmitted at the $k$-th time slot, the received pilot signal is given as

$$
y_{k}=h_{k} a_{k} s_{k} g_{k} x+n_{k}
$$

From (10), the estimate of the cascaded channel coefficient related to the $k$-th IRS unit is computed as

$$
\hat{h}_{k} \hat{g}_{k}=y_{k}\left(a_{k} s_{k} x\right)^{-1} \text {. }
$$

In this approach, the cascaded channel estimation is accomplished after $K$ time slots. The ON/OFF channel estimation method is summarized in Algorithm 1.

\section{B. IRS Optimization}

After the channel estimation using Algorithm 1, the phase shifts at the IRS are designed to maximize the signal to noise ratio (SNR) at the receiver during the data transmission phase. The SNR is maximized by "aligning" the IRS phase shifts to the channels phase such that they add coherently at the receiver. The optimal choice for the continuous phase shift associated with the $k$-th IRS unit is computed as [12],

$$
\phi_{k}^{\text {(opt) }}=-\angle \hat{h}_{k} \hat{g}_{k}, \quad \forall k=1, \ldots, K .
$$




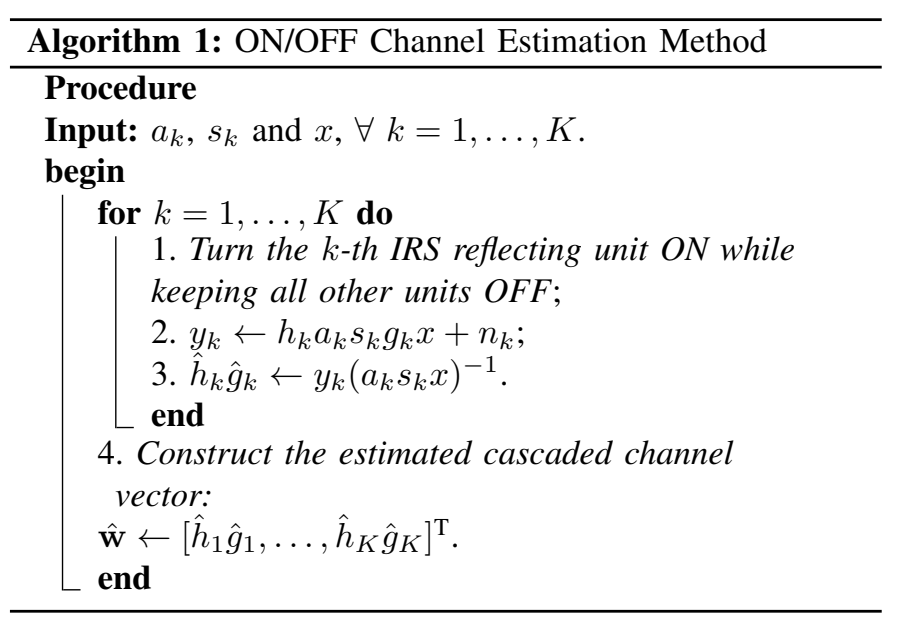

The discretized phase shift corresponding to (12) can be obtained as follows

$$
\bar{\phi}_{k}^{(\mathrm{opt})}=\min \left|\phi_{k}^{(\mathrm{opt})}-f \cdot \Delta \phi\right|, f=1, \ldots, F-1 .
$$

\section{NUMERICAL RESUlts}

In this section, the performance of the IRS-assisted wireless communication system is evaluated under different kinds of practical impairments. The impact of the varactor model on the channel estimation accuracy is evaluated in terms of the normalized mean square error (NMSE) between the true and the estimated cascaded channels as

$$
\operatorname{NMSE}(\hat{\mathbf{w}})=\frac{1}{T} \sum_{\tau=1}^{T}\left(\frac{\left\|\mathbf{h} \odot \mathbf{g}-\hat{\mathbf{w}}_{(r)}\right\|_{\mathrm{F}}^{2}}{\|\mathbf{h} \odot \mathbf{g}\|_{\mathrm{F}}^{2}}\right),
$$

where $\hat{\mathbf{w}}_{(\tau)}=\hat{\mathbf{h}} \odot \hat{\mathbf{g}}$ is the cascaded channel estimated at the $\tau$-th Monte Carlo run. The spectral efficiency is calculated as

$$
\mathrm{SE}=\log _{2}\left(1+\frac{\left|\mathbf{g}^{\mathrm{T}} \operatorname{diag}(\mathbf{e} \odot \mathbf{a} \odot \mathbf{s}) \mathbf{h}\right|^{2}}{\sigma^{2}}\right) .
$$

Note that the spectral efficiency formula considers the general signal model in (2) and perfect channel knowledge. When considering the particular cases of this signal model, the IRSrelated terms e, a, s are adjusted accordingly. The performance curves are plotted as a function of the transmitted signal energy to noise density ratio $\left(E_{b} / N_{0}\right)$ for an IRS of $K=100$ units. Our results represent an average over $T=10^{4}$ independent Monte Carlo runs. Besides, the considered varactor hardware model is configured with the parameters given in Table I of Section II-C, the phase shift model (9), and $R_{k}=1 \Omega$. For the discrete-phase model, we assume $b=4$ bits, yielding $2^{4}=16$ levels of quantization.

In Figure 4, we compare the performance of the models presented in Section II. The idealized IRS model assumes an infinite phase shift resolution and unitary amplitude response, while the discrete model assumes quantized phase shifts and fluctuations in the amplitude response. In addition, we also plot the practical case considering the varactor-based (physical) model, where the phase is based on Figure 3. For both the discrete and the varactor models, the phase shifts are quantized according to the minimum distance criterion on the set $\mathbb{F}$

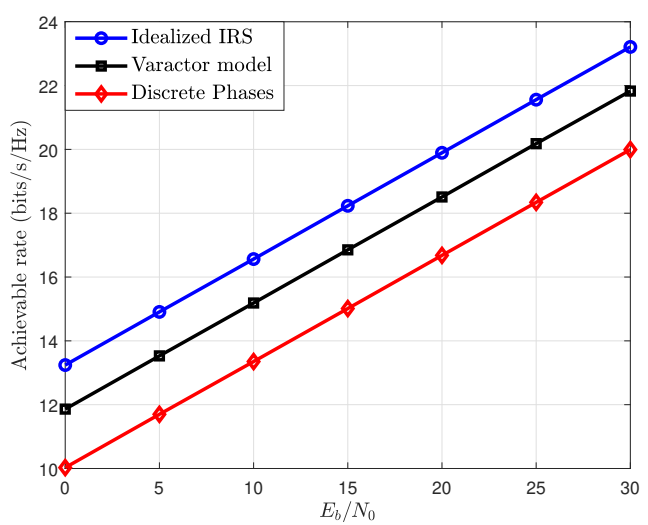

Fig. 4. Spectral efficiency by considering the effect of the practical hardware model and discrete phase shift.

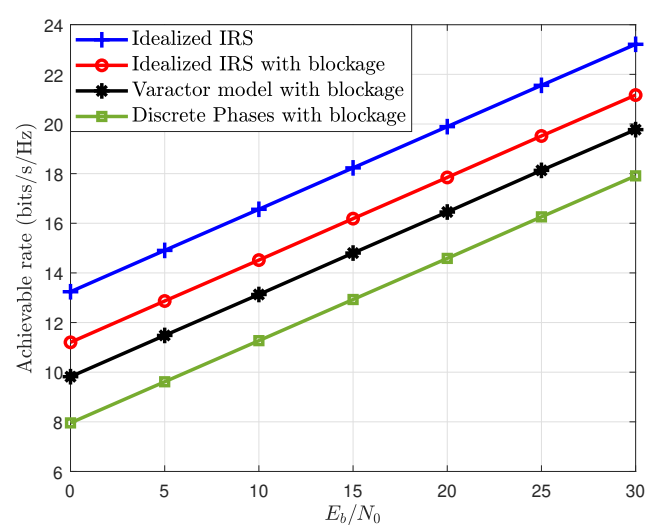

Fig. 5. Spectral efficiency by considering blockage effect in the IRS reflecting units, practical hardware model and discrete phase shift.

constructed as shown in Section II-B. As we can see in Figure 4 , the varactor model has a performance gap of approximately $5 \mathrm{~dB}$ when compared to the idealized IRS model, while the discrete phase shift model presents the worse results.

Figure 5 complements the result in Figure 4, analyzing the impact of blockages affecting IRS units in terms of spectral efficiency. In this experiment, we assume that $P_{B}=50 \%$, i.e., half of the IRS units are unavailable due to blockages. We observe that the idealized case outperforms the varactorbased model also when IRS units are blocked, which is an expected result. In Figures 4 and 5, we can see that the varactor model overcomes the discrete phase model, because in the first one the amplitude responses are limited to the interval $0.5774<a_{k}<0.9991$ (according to Figure 3), while in the discrete model the amplitude responses are uniformly distributed within the interval $0<a_{k}<1$ and are not physically coupled to the phase shift responses. Therefore, we can note that the discrete phase shift model is pessimistic in capturing more aggressive amplitude fluctuations, indicating a high power dissipation compared to the varactor model and reducing its spectral efficiency. These result corroborate the importance of considering a physical hardware-based model for the IRS, such as the one based on varactor circuits, since it provides a more concrete and realistic view of the practical implementation of an IRS-assisted communication system.

In Figure 6, we evaluate the impact of the varactor model 


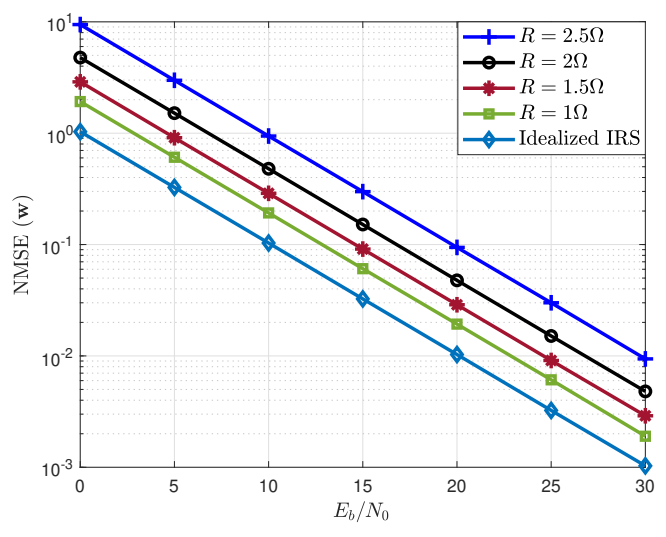

Fig. 6. NMSE of the estimated cascaded channel $\hat{\mathbf{w}}$.

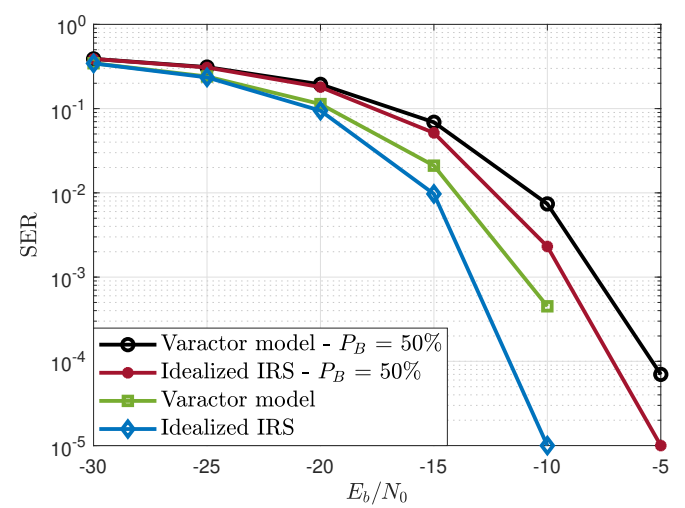

Fig. 7. SER performance of the idealized and varactor model approaches.

on the channel estimation performance. We plot the NMSE curves for different resistance values. It becomes evident that increasing the resistance of the varactor model leads to an increasing absorption (or energy dissipation), limiting the channel estimation performance. By comparing the result that assumes $R_{k}=2.5 \Omega$ with the one assuming $R_{k}=1 \Omega$, we note a performance degradation of approximately $7 \mathrm{~dB}$, showing that the varactor model is more efficient when it operates under low resistance values. In order to reduce the resistance values, manufacturers usually employ an epitaxial structure in their design, choosing $R_{k}$ according to the breakdown voltage function of the used diode [16]. It is important to mention that the degradation in the channel estimation performance can always be observed when varactor models are considered, since $R_{k}=0$ is unfeasible. Indeed, some power dissipation at the P-N junction always exist.

In Figure 7, we evaluate the symbol error rate (SER) performance assuming BPSK data symbols. The IRS phase shifts are optimized from the estimated cascaded channel. We compare the idealized IRS model and the practical varactorbased model by assuming IRS units operating with and without blockages. We can observe that the idealized model and the varactor model showed a very close SER performance. However, in the presence of blockages, a performance degradation is observed as the $E_{b} / N_{0}$ increases. This suggests that at high $E_{b} / N_{0}$ regime, symbol detection becomes more sensitive to the presence of blockage impairments.

\section{CONCLUSiON}

We have studied the impact of IRS non-idealities and channel estimation errors on the IRS-assisted SISO communication performance. For this purpose, we developed a signal model that unifies different kinds of realistic impairments on the IRS design, such as physical coupling between amplitudes and phases in the varactor hardware model, discrete phase shift resolution and the unavailable of some IRS reflecting units due to any kind of inevitable blockages. Simulation results have shown that, compared with the idealized IRS model without amplitude, phase and blockage impairments, hardware nonidealities degrade the system performance and should be taken into account when evaluating practical systems. Perspectives include the expansion of this analysis to the MIMO scenario.

\section{REFERENCES}

[1] Y. Liu, X. Liu, X. Mu, T. Hou, J. Xu, M. Di Renzo, and N. Al-Dhahir, "Reconfigurable intelligent surfaces: Principles and opportunities," IEEE Commun. Surveys Tuts, pp. 1-1, 2021.

[2] M. Hua, L. Yang, Q. Wu, C. Pan, C. Li, and A. Lee Swindlehurst, "UAV-assisted intelligent reflecting surface symbiotic radio system," IEEE Trans. Wireless Commun., pp. 1-1, 2021.

[3] N. C. Luong, T. T. V. Nguyen, S. Feng, H. T. Nguyen, T. D. Niyato, and D. I. Kim, "Dynamic network service selection in IRS-assisted wireless networks: A game theory approach," IEEE Trans. Veh. Technol., pp. 1-1, 2021.

[4] S. Xu, Liu, Jiajia, Y. Cao, J. Li, and Y. Zhang, "Intelligent reflecting surface enabled secure cooperative transmission for satellite-terrestrial integrated networks," IEEE Trans. Veh. Technol., vol. 70, no. 2, pp. 2007-2011, 2021.

[5] M. Toumi and A. Aijaz, "System performance insights into design of RIS-assisted smart radio environments for 6G," in IEEE Wireless Commun. and Netw. Conf., 2021, pp. 1-6.

[6] T. L. Jensen and E. De Carvalho, "An optimal channel estimation scheme for intelligent reflecting surfaces based on a minimum variance unbiased estimator," in IEEE Int. Conf. on Acoust., Speech and Signal Proc., 2020, pp. 5000-5004.

[7] Q. Wu and R. Zhang, "Beamforming optimization for wireless network aided by intelligent reflecting surface with discrete phase shifts," IEEE Trans. Commun., vol. 68, no. 3, pp. 1838-1851, 2020.

[8] G. T. de Araújo, L. C. de P. Pessoa, and A. L. F. de Almeida, "Channel estimation for MIMO system assisted by intelligent reflective surface," XXXVIII Brazilian Symposium on Telecommunications and Signal Processing, 2020.

[9] B. Li, Z. Zhang, Z. Hu, and Y. Chen, "Joint array diagnosis and channel estimation for RIS-aided mmwave MIMO system," IEEE Access, vol. 8, pp. 193992-194 006, 2020.

[10] S. Abeywickrama, R. Zhang, Q. Wu, and C. Yuen, "Intelligent reflecting surface: Practical phase shift model and beamforming optimization," IEEE Trans. Commun., vol. 68, no. 9, pp. 5849-5863, 2020.

[11] J.-B. Gros, V. Popov, M. A. Odit, V. Lenets, and G. Lerosey, "A reconfigurable intelligent surface at mmWave based on a binary phase tunable metasurface," IEEE Open J. of the Commun. Soc., pp. 1-1, 2021.

[12] E. Basar, M. Di Renzo, J. De Rosny, M. Debbah, M.-S. Alouini, and R. Zhang, "Wireless communications through reconfigurable intelligent surfaces," IEEE Access, vol. 7, pp. 116753-116773, 2019.

[13] X. Ma, Z. Chen, W. Chen, Y. Chi, L. Yan, C. Han, and S. Li, "Joint hardware design and capacity analysis for intelligent reflecting surface enabled terahertz MIMO communications," 2020, arXiv:2012.06993v1.

[14] Y. Yang, B. Zheng, S. Zhang, and R. Zhang, "Intelligent reflecting surface meets ofdm: Protocol design and rate maximization," IEEE Trans. Commun., vol. 68, no. 7, pp. 4522-4535, 2020.

[15] B. O. Zhu, J. Zhao, and Y. Feng, "Active impedance metasurface with full $360^{\circ}$ reflection phase tuning," Scientific reports, vol. 3, pp. 30593064, 2013.

[16] T. Lee, "Evaluation of voltage dependent series resistance of epitaxial varactor diodes at microwave frequencies," IEEE Trans. Electron Devices, vol. 12, pp. 457-470, 1965. 